Meta

Journal des traducteurs

Translators' Journal

\title{
Initiation à l'interprétation consécutive à l'Institut national des langues et civilisations orientales à Paris
}

\section{Daniel Gile}

Volume 27, numéro 3, septembre 1982

URI : https://id.erudit.org/iderudit/002889ar

DOI : https://doi.org/10.7202/002889ar

Aller au sommaire du numéro

Éditeur(s)

Les Presses de l'Université de Montréal

ISSN

0026-0452 (imprimé)

1492-1421 (numérique)

Découvrir la revue

Citer cette note

Gile, D. (1982). Initiation à l'interprétation consécutive à l'Institut national des langues et civilisations orientales à Paris. Meta, 27(3), 347-351.

https://doi.org/10.7202/002889ar d'utilisation que vous pouvez consulter en ligne. 
INITIATION À L'INTERPRÉTATION CONSÉCUTIVE À L'INSTITUT NATIONAL DES LANGUES ET CIVILISATIONS ORIENTALES À PARIS

Les études de japonais à l'INALCO mènent au Diplôme de japonais en deux ans, à la licence

19. Maurice-Edgar Coindreau a dédié ses Mémoires d'un traducteur (Paris, Gallimard, 1974) à «M Mie Guénon qui, à la fin du siècle dernier, au lycée de la Roche-sur-Yon, [lui] apprit à lire et à écrire». 
ou au Diplôme supérieur en trois ans, puis à la maîtrise et au doctorat.

En troisième année, les étudiants choisissent entre trois options de spécialisation: "langue et littérature », «relations internationales » et «interprétation et traduction».

Il existe à l'Institut depuis plus de dix ans une unité de valeur annuelle intitulée «initiation aux techniques de l'interprétation », obligatoire en «interprétation et traduction» et facultative en «relations internationales». Par ailleurs, une autre unité de valeur de troisième année, «étude de textes économiques», comporte une séance hebdomadaire d'exercices d'interprétation. Une UV de quatrième année, «entraînement aux techniques de l'interprétation», complète la série de cours consacrés à cette discipline.

Jusqu'en 1980, l'UV «initiation aux techniques de l'interprétation» était entièrement vouée à des sujets économiques. Les séances, hebdomadaires, se déroulaient en laboratoire de langues et duraient trois heures. Durant la première moitié du cours, l'enseignant passait en revue les principales idées d'un petit texte économique sélectionné dans un livre scolaire japonais et préparé par les étudiants, puis expliquait certains termes et tournures japonais susceptibles de poser des difficultés de compréhension à l'écoute.

Puis se déroulait l'exercice d'interprétation proprement dit: l'enseignant faisait passer sur le pupitre central l'enregistrement d'un texte lu en japonais, se composant de quatre parties d'une minute environ séparées par des «blancs» d'une durée équivalente, et paraphrasant ou commentant le texte économique préparé. Les étudiants écoutaient la cassette dans leur casque individuel en prenant des notes et enregistraient leur interprétation de chaque passage pendant le «blanc» qui le suivait. Puis, les volontaires «rendaient» leur cassette qui était écoutée en classe et commentée par l'enseignant.

Les travaux dirigés de l'UV «étude de textes économiques» se déroulaient selon des modalités analogues, à cela près que les séances ne duraient qu'une heure et demie et que la cassette préparée par l'enseignant se composait d'un enregistrement continu de plusieurs minutes, et non pas de plusieurs sections séparées par des «blancs".

En 1980 a été tentée une transformation de l'UV «initiation aux techniques de l'interprétation" dans le sens d'une pédagogie plus axée sur les principes théoriques et les aspects pratiques de l'interprétation consécutive dans une optique professionnelle que sur le perfectionnement linguistique en japonais.

Le principal obstacle méthodologique à franchir était précisément d'ordre linguistique. En début de troisième année, les étudiants n'ont en général à leur actif que deux années d'étude du japonais. Ils ont donc acquis un vocabulaire élémentaire et une certaine familiarité avec les structures syntaxiques et les tournures les plus fréquentes du japonais écrit, mais sont infiniment loin du niveau de compréhension requis. En effet, dans le classement linguistique en vigueur à l'AIIC (Association Internationale des Interprètes de Conférence), même la langue " $C$ ", langue passive par opposition à la langue « $\mathrm{B} »$ (active) et à la langue «A» (maternelle) «... doit être comprise à l'audition... de façon aussi complète et précise que les $\approx A$ » et $« B$ * ... la langue $A$ étant une langue maternelle cultivée, dans laquelle le candidat possède une grande richesse de vocabulaire et qu'il doit savoir manier avec précision et aisance $» 1$.

En fait, la disparité entre les connaissances linguistiques requises et le cas des effectifs de troisième année à l'INALCO était telle qu'elle faisait douter de la possibilité d'enseigner l'interprétation à ces étudiants, chez qui le passage de l'énoncé linguistique au message était si laborieux et si incomplet.

Toutefois, l'interprétation repose sur l'écoute attentive et l'exégèse des paroles de l'orateur, éventuellement sur une méthode de prise de notes, et sur un effort de clarté et de persuasion dans la ré-expression du message, trois éléments qui ne sont pas essentiellement linguistiques, mais intellectuels. Il nous a semblé que l'expérience pédagogique méritait d'être tentée, quitte à employer un japonais artificiellement simplifié.

Il fallait donc trouver une formule permettant de préparer un discours linguistiquement simple mais présentant d'une part le caractère d'un discours spontané, et d'autre part une densité et une richesse d'informations suffisantes pour demander un véritable effort d'écoute et d'exégèse.

Pour des raisons que l'on trouvera très bien exposées dans un récent travail de Karla DEJEAN - LE FEAL de l'ESIT ${ }^{2}$, la lecture d'un texte écrit ne répondait pas aux critères méthodologiques. D'un autre côté, la présentation en classe de discours véritablement spontanés dès le début de l'année était susceptible de poser des difficultés de compréhension telles que l'exercice serait mis en échec. On a donc opté pour la préparation d'une cassette dont l'enregistrement pourrait être réalisé de manière à obtenir un produit fini satisfaisant à soumettre aux étudiants.

Il s'agissait essentiellement d'interviews avec des questions en français et des réponses

1. Extrait de la brochure d'information de l'ESIT destinée aux candidats à la section Interprétation.

2. Il s'agit d'une thèse de doctorat de troisième cycle soutenue par K. DEJEAN à Paris en 1978 et intitulée «Lectures et improvisations». Cf. Meta, vol. 26, n 3, p. 300-302. 
en japonais. Chacune des réponses était écoutée et ré-enregistrée si elle contenait des mots ou tournures menaçant sérieusement la compréhension du message. Toutefois, les incidents de parcours propres à l'expression spontanée, telles que les variations de rythme, redites, hésitations, redondances et maladresses ne faisaient l'objet d'aucune correction.

Quant à la progression des thèmes des interviews, elle était guidée par des considérations didactiques, par la recherche d'une certaine variété et par l'évolution dans le sens d'une technicité croissante.

Les premiers exercices étaient conçus de manière à récapituler, compléter et illustrer les prircipes de l'interprétation tels que présentés par les enseignants en guise de préambule, puis dans les commentaires sur la prestation des étudiants. Il s'agissait de l'«interview» d'un interprète japonais expliquant la nécessité de comprendre pour traduire, les similitudes et différences entre traduction et interprétation, la prise de notes, l'importance de la recherche documentaire. Les commentaires des enseignants, en français et en japonais, se substituaient à ce premier stade à celle-ci, et fournissaient les éléments linguistiques nécessaires.

Cette prise de contact avec l'interprétation était suivie d'une série d'exercices sur le tourisme japonais, sujet ne présentant aucune difficulté de fond mais comportant un vocabulaire propre et sélectionné notamment pour attirer l'attention des étudiants sur l'intérêt de la préparation linguistique.

Enfin, à un troisième stade, intervenaient des sujets de plus en plus techniques et nécessitant une véritable recherche documentaire et terminologique: alpinisme, économie, microélectronique, etc.

\section{Déroulement des cours}

Les séances de travail se déroulaient dans une salle simple plutôt qu'en laboratoire, et les étudiants écoutaient la cassette enregistrée à travers le haut-parleur d'un magnétophone. Les exercices d'interprétation démarraient tout de suite, le travail préparatoire étant censé avoir été accompli hors-cours. Pour cela, les étudiants disposaient d'une liste de mots qui leur était communiquée une semaine à l'avance, de dossiers contenant articles et documents en français et en japonais mis à leur disposition à la bibliothèque, et de cassettes enregistrées permettant un travail préparatoire individuel en laboratoire.

L'enseignant passait la question de l'intervieweur et une partie ou la totalité de la réponse de l'interviewé et demandait à un, deux ou trois étudiants d'en assurer l'interprétation. Les autres étudiants critiquaient et complétaient, et l'enseignant ajoutait ses propres commentaires spécifiques et généraux.
Quelques exercices spéciaux, toutefois, le premier lors de la séance initiale de l'année, et les autres au cours des deuxième et troisième trimestres, étaient prévus.

Le tout premier exercice de l'année s'inspirait de la démarche de l'ESIT et visait à sensibiliser les étudiants à des difficultés invisibles pour le profane mais fondamentales, par le biais d'un exercice français-français, l'«interprétation" d'un petit article du Monde, lu à faible vitesse ${ }^{3}$. Cette démonstration a permis de mettre en relief les phénomènes suivants :

1. L'impression de comprendre un discours peut être tout à fait illusoire, comme le montraient les contradictions et incohérences relevées chez les étudiants.

2. L'écoute attentive ne suffit pas toujours à mémoriser la teneur d'un discours.

3. En situation d'écoute «normale», des «trous» peuvent se produire. Nombreux étaient les étudiants qui se sont étonnés de constater qu'ils n'avaient pas «entendu " certains éléments du discours, en dépit de leur concentration.

À la différence de l'ESIT, cette démonstration n'a pu être suivie d'exercices de "mémorisation ", et les principes de la prise de notes ont été abordés séance tenante.

Par ailleurs, au cours de la deuxième moitié de l'année, différentes expériences ont été tentées:

1. Interprétation d'exposés spontanés faits en classe par les étudiants japonais. Malheureusement, les obstacles linguistiques se sont avérés excessifs, et l'expérience a dû être abandonnée.

2. Interprétation par les Japonais de discours spontanés français. Cet exercice était destiné à permettre aux Japonais de travailler vers leur langue maternelle et aux Français de mettre leurs techniques d'écoute et de prise de notes à l'épreuve d'un discours ne présentant aucune difficulté linguistique.

\section{Enseignement théorique}

Le cours ne comportait aucune partie distincte consacrée à la linguistique. Le seul élément véritablement abstrait de l'enseignement était l'explication du non-isomorphisme entre langage et pensée, nécessaire pour justifier l'emploi, dans la langue d'arrivée, de mots et de structures non parallèles à ceux du discours de l'orateur. Les autres considérations théoriques étaient directement liées à la pratique et intervenaient à la suite des exercices et des questions

3. Ce choix laisse pourtant à désirer : si la richesse et la densité du texte assurent la réussite de la démonstration, elles représentent les caractéristiques de la langue écrite, et non pas du discours spontané. Le recours à l'enregistrement d'un discours suffisamment dense serait méthodologiquement plus satisfaisant. 
des étudiants. Il s'agissait essentiellement de la justification des principes de la stratégie interprétative : comment agir en cas de débit trop rapide de l'orateur, si l'on "manque" une idée, comment interpréter un discours très violent, des jeux de mots, des citations, etc.

Le processus commençait en général par une constatation, faite lors d'un exercice, et qui donnait lieu à une question de la part des étudiants. Les explications étaient données à titre ponctuel, sans tentative de rattachement des divers éléments à une "théorie de la traduction", mais sous le signe de la communication, but ultime et raison d'être de l'interprétation. Elles étaient parfois accompagnées d' «expériences" démontrant le bien-fondé de certaines affirmations et pratiques, notamment par le biais des groupes témoins. Les étudiants ont pu ainsi constater l'utilité de la recherche documentaire (interprétation du même discours par un groupe familiarisé avec le sujet et un autre qui ne l'était pas), l'effet perturbateur de la prise de notes sur l'écoute, l'efficacité des différentes méthodes de prise de notes, etc.

\section{Interaction étudiants-enseignants}

Il nous a semblé important, pour obtenir une plus forte motivation dans un exercice difficile, pour mieux convaincre, et surtout pour permettre aux étudiants de projeter leur personnalité dans leur interprétation, de s'écarter du modèle classique des rapports verticaux enseignantsenseignés en faveur d'une formule plus égalitaire.

Des efforts ont donc été déployés en vue d'atténuer le caractère habituellement proéminent des éléments proprement scolaires tels que notes, examens et affirmations magistrales, tout en favorisant la participation, et notamment les commentaires et critiques des étudiants à l'égard des réflexions des enseignants.

Après une période initiale de mise en confiance, le dialogue s'est engagé, ce qui a permis entre autres de relever et de tenter de corriger certaines faiblesses dans les argumentations et démonstrations des enseignants. En outre, la progression et les difficultés de chacun ont pu être suivies d'assez près.

\section{Évaluation des résultats}

D'une manière générale, les éléments théoriques et principes stratégiques semblent avoir été acceptés et bien assimilés. Des commentaires incompris en début d'année ont progressivement été appréhendés avec davantage de précision, et un relief des priorités s'est dégagé, permettant aux étudiants de saisir la gravité d'un petit écart de nuance par rapport à une grande maladresse sémantique, l'importance de la charge diplomatique des mots et le rôle $\mathrm{du}$ choix du registre linguistique dans la communication.
Dans un autre domaine, l'expression verbale des étudiants s'est sensiblement améliorée durant l'année et la récitation souvent hésitante du début a cédé la place dans la plupart des cas à un discours plus assuré et plus posé.

Mais e'est dans le domaine de la prise de notes que les observations les plus intéressantes ont pu être faites. Dans ce domaine, les enseignants s'étaient bornés à expliquer que les notes ne devaient constituer que des béquilles pour aider la mémoire et qu'elles étaient voraces en énergie aux dépens de l'écoute, sans proposer de véritable système. Or, la plupart des étudiants ont opté rapidement pour des notes brèves, à base de Kanji (caractères chinois), avec séparation des idées, décalage et verticalité.

Ces principes figurent précisément parmi les règles d'or de la prise de notes en consécutive telle que généralement pratiquée par les interprètes de conférence professionnels ${ }^{4}$.

Précisons que les Kanji permettent d'évoquer les idées plutôt que des mots, que, contrairement aux idées reçues, ils s'écrivent vite, et que même mal écrits, ils sont aisément identifiables en contexte grâce à leur forme générale.

Globalement parlant, les prestations de la majorité des étudiants étaient sérieuses, assez complètes, et surtout intelligentes, prouvant ainsi que l'interprétation avait été COMPRISE. Mais a-t-elle également été APPRISE et maitrisée comme dans les écoles spécialisées?

La question est justifiée, puisque rien ne prouve a priori l'identité des efforts entrant un jeu dans le traitement d'un discours linguistiquement simple et peu dense et des opérations mentales qui interviennent dans l'interprétation d'un discours plus dur. L'épreuve d'un exercice ardu aurait peut-être débouché sur un échec.

Le niveau linguistique insuffisant des effectifs de troisième année rend la vérification directe impossible. Toutefois, une évaluation de la capacité acquise dans la partie «muette» de l'interprétation (écoute, analyse, prise de notes) serait envisageable dans le cadre d'un exercice de consécutive français-français, dense et technique à souhait.

\section{Conclusion}

Les cours d'interprétation dispensés dans les écoles spécialisées, universités et autres établissements sont nombreux, mais le détail des démarches, techniques et méthodes utilisées reste largement inconnu, faute d'un échange d'informations suffisant.

Nul ne le contestera: devenir un véritable interprète, c'est travailler sans relâche à parfaire ses connaissances linguistiques, générales et techniques, c'est se familiariser avec

4. Voir par exemple Jean-François ROZAN (1974): la Prise de notes en interprétation consécutive, Genève, Librairie de l'Université Georg. 
la psychologie des orateurs et avec leurs procédés, c'est acquérir consciemment et volontairement une vaste expérience sur le terrain au fil des mois et des années.

Mais la préparation du premier envol, possible dès que l'interprète débutant dispose, outre des connaissances linguistiques de base, d'une maîtrise opérationnelle des stratégies fondamentales, pourrait être optimisée de manière à faire gagner beaucoup de temps pour des résultats précis.

Dans cette optique, les échanges d'information entre enseignants, notamment par le biais d'articles tels que celui-ci, sont susceptibles d'apporter une contribution importante en permettant à tous de profiter des initiatives de chacun.
Note: l'enseignement a été assuré en 1980/81 par une équipe de deux enseignants, l'auteur, francophone, et N. ROLAND, de langue maternelle japonaise.

Daniel Gile 